Depuis que I'approbation d'une rémunération forfaitaire des prestations hospitalières par les chambres fédérales ne fait plus de doute, I'introduction de la structure tarifaire SwissDRG est devenue inéluctable. Suivant les cantons, un nombre significatif d'hôpitaux ont cependant déjà subi la mise en application d'un système DRG, parfois depuis plusieurs années. Les expériences ainsi accumulées ont permis aux utilisateurs concernés de déceler certains points faibles du système, d'en mesurer les conséquences et de développer aussi des solutions adaptées à leur problème particulier. Celles-ci étant susceptibles d'intéresser tous les médecins actifs à I'hôpital, c'est avec plaisir que nous publions I'article ci-dessous.

La rédaction

\title{
Von der medizinischen Dokumentation zur DRG-Abrechnung
}

Grundlage für die Abrechnung und somit für die Finanzierung mit DRGs ist eine qualitativ hochwertige Dokumentation des behandelnden Arztes und der Pflege. Jede Dokumentation enthält eine Fülle medizinischer und administrativer Informationen in unterschiedlich aufbereiteter Form. Zu nennen sind Rohdaten aus dem Kardex, Formulare und Vordrucke. Hinzu kommen die für den Kodierer wichtigsten Dokumente wie Austritts- und OP-Berichte, Konsile und Befunde. Sie stellen zusammenfassende Dokumente für die Kodierung und somit Abrechnung über APDRG dar. Idealerweise können diese von den Kodierkräften zum Abgreifen der ICD- und CHOP-Kodes für die Kodierung herangezogen werden. Nur in seltenen Fällen sollte ein Nachschlagen in der kompletten Krankengeschichte erforderlich sein. Als Ergebnis kann eine richtige DRG kodiert und eine korrekte Rechnung gestellt werden. Als Ziel darf neben einer korrekten Kodierung aufgrund präziser medizinischer Dokumentation die administrative Entlastung der ärztlichen Leistungserbringer durchaus oberste Prämisse sein.

\section{Rolf Malk ${ }^{a}$, Gerhard Daum ${ }^{b}$}

a Geschäftsführer, Malk Medizin Controlling AG b Dr. med., Arzt und Medizincontroller in der Schweiz
Korrespondenz:

Rolf Malk

Malk Medizin Controlling AG

Alpenblickstrasse 20

CH-8853 Lachen SZ

Tel. 0554122516

Fax 0554122564

malk@medizincontrolling.ch

www.medizincontrolling.ch

\section{Einleitung}

Mit der Einführung der DRGs werden von verschiedenen Institutionen hohe und teilweise auch völlig neue Anforderungen an die stationäre Versorgung gestellt. Aus Sicht des Gesetzgebers führt die Einführung der leistungsorientierten pauschalisierten Abrechnung mit SWISS-DRGs (zur Zeit noch APDRGs) zu einer möglichen Transparenz im Spitalsektor als Voraussetzung, um in diesem Markt steuernd einzugreifen. Transparenz entsteht einerseits durch die uns bereits bekannte Anwendung der Klassifikationssysteme ICD und CHOP und der vorwiegend administrativen Patientendaten wie Alter, Geschlecht, Aufenthaltsdauer und Austrittsart. Andererseits beinhalten die DRGs neue Kennzahlen wie Kostengewichte, CaseMix, Case-Mix-Index, untere, mittlere und obere Aufenthaltsdauer (LTP, ALOS, HTP), die zu einer weiteren Transparenz im Leistungsgeschehen führen. Für die sachgerechte Generierung einer DRG benötigt der Kodierer umfangreiche Informationen, um diagnostischen, therapeutischen und pflegerischen Aufwand des zur Abrechnung stehenden stationären Aufenthaltes korrekt abbilden zu können. Beim «Kodierer» kann es sich sowohl um den kodierenden Arzt als auch um einen eigens eingestellten Kodierer handeln.

\section{Vision}

Mit Hilfe von ausgereiften Softwaretools soll die Papierdokumentation in eine überwiegend 
«elektronische» Krankengeschichte (KIS/SIS) überführt werden, die allen Anforderungen einer modernen und zukunftsorientierten Datenaufbereitung gerecht wird. Somit kann eine Vereinheitlichung der medizinischen Dokumentation und vor allem eine Erleichterung der dokumentierenden und behandelnden Ärzteschaft in «greifbare Nähe» rücken. Neben der erforderlichen Ausrüstung (Hard- und Software) ist für die Umsetzung auch eine entsprechende Schulung aller Prozessbeteiligten (Arzt, Pflege, Kodierkraft) erforderlich.

Der Dokumentationsaufwand soll im Sinne aller Leistungserbringer vereinfacht und die Berichtsstruktur über alle Abteilungen standardisiert werden. Die am Gesamtprozess beteiligten Einheiten wie Arzt, Pflege, Labor, Diagnostik, Kodierer, EDV und Patientenverwaltung sollen einen möglichst geringen administrativen Aufwand haben. Synergieeffekte ergeben sich vor allem durch eine Standardisierung im gesamten Spital.

\section{Gegenwart}

Aus Sicht «Kodierung und somit Fakturierung» führen wenig präzis formulierte Diagnosen oder zum Teil unvollständige Auflistungen von Diagnosen in den zusammenfassenden Berichten (wie zum Beispiel dem Austrittsbericht) durch das Suchen in den Rohdaten zu einem erhöhten Aufwand in der Kodierung. Zusätzlich sind sie auch Ursache für das «Nichtfinden» wichtiger kodierrelevanter Informationen. Als Ergebnis können DRGs mit Ertragseinbussen generiert werden.

Besondere Aufmerksamkeit gilt deshalb der korrekten, vollständigen und für die Kodierung vor allem «leichtverständlichen» Dokumentation. Potentielle Schwachstellen in den unterschiedlichen Ebenen des Dokumentationsablaufes (Krankengeschichte, Berichtswesen, Kodierung) müssen im Vorfeld erkannt und minimiert werden. Denn das Endergebnis - die DRG - kann immer nur so gut (sachlich korrekt) sein, wie die Ausgangsdaten (Krankengeschichte und zusammenfassende Berichte) dies auch hergeben. Das heisst, die Qualität der Kodierung (und damit Fakturierung) fängt immer bei der Dokumentation an!

Jede Krankengeschichte enthält eine Fülle von medizinischen und administrativen Informationen in unterschiedlich aufbereiteter Form und aus unterschiedlichen Gründen (Informationen nachbehandelnder Institutionen, Archivierung, juristische Absicherung - und neu eben Kodierung und Fakturierung). Zu nennen sind die Pflegedokumentation (Kardex) und die vie-

\section{De la documentation médicale à la facturation DRG}

Les documents du médecin traitant et des soins infirmiers servant de base à la facturation, et donc au financement par le système DRG, doivent être de haute qualité. Chaque dossier contient une foule d'informations médicales et administratives présentées sous différentes formes. Mentionnons les données brutes provenant du cardex, les formulaires et les préimprimés mais aussi les rapports d'opération et d'examens spécialisés ainsi que les lettres de sortie d'hôpital qui sont des documents récapitulatifs de première importance pour la codification et la facturation par les groupes APDRG, desquels on obtient les codes CIM et CHOP. La possibilité de consulter le dossier médical complet ne devrait être requise que dans de rares cas. On parviendra ainsi à codifier le groupe DRG correct et à établir une facturation tout aussi correcte. Outre le fait de pouvoir établir une codification correcte sur la base de documents médicaux établis avec précision, on atteindra un autre objectif important, celui d'alléger les tâches administratives des fournisseurs de prestations médicales.

len unterschiedlichen Formulare für den ärztlichen und pflegerischen Bereich. Diese Dokumente stellen die Rohdaten der Krankengeschichte dar. Hinzu kommen die für den Kodierer so wichtigen Austrittsberichte, OPBerichte, Verlaufsprotokolle und Befunde. Sie stellen zusammenfassende Dokumente dar.

Häufig enthalten diese Dokumente nicht alle Informationen, die die Kodierkraft zum Abgreifen der Daten (ICD und CHOP) benötigt. Ein Nachschlagen und Suchen in den Rohdaten (Krankengeschichte) ist deshalb oftmals erforderlich.

\section{Anforderungen an die medizinische Dokumentation}

Auch die nichtelektronisch geführten Teile der Krankengeschichte müssen für Dritte gut leserlich ausgefüllt werden. Diagnostik, Therapie und Verlaufkontrolle müssen für Dritte erkennbar sein. Die verwendeten Formulare sollten (auch 
im Sinne einer Verhinderung von «zuviel» Dokumentation für den Leistungserbringer) auf ein Mindestquantum reduziert werden. Der Aufbau der gesamten Krankengeschichte sollte mittels eines klinikspezifischen und einheitlichen Inhaltsverzeichnisses standardisiert werden. Jeder Spitalaufenthalt sollte in einer Akte abgelegt werden bzw. durch Trennblätter klar abgegrenzt sein. Denn DRGs beziehen sich immer nur auf einen einzigen Spitalaufenthalt. So können Verwechslungen beim Abgreifen für die Kodierung vermieden werden. Zur Sicherheit können sämtliche Dokumente mit dem Patientenetikett beschriftet werden. Dies führt zur Vermeidung von nicht zuordenbaren losen Blättern. Jedes Dokument sollte nur einfach in der «Akte» enthalten sein. Mehrfachkopien führen zur Unübersichtlichkeit. Sämtliche Dokumente sollten in fest gebundener Form vorliegen, nur dann ist die Akte vor Informationsverlust geschützt.

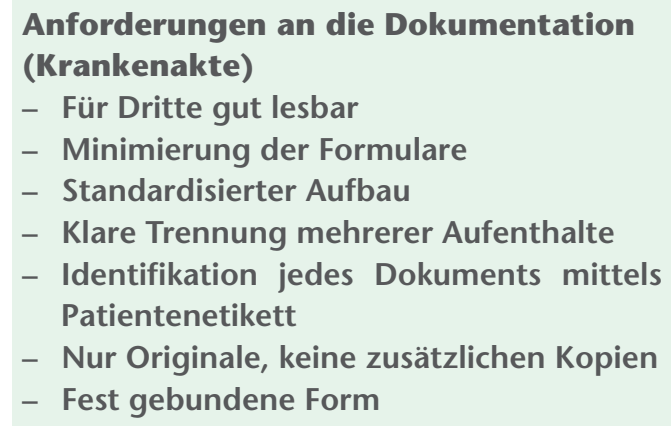

\section{Anforderungen an die Ärzte}

Neben der klinischen Terminologie sollten die behandelnden Ärzte (ganz gleich, ob sie «kodierende Ärzte» oder «nichtkodierende Ärzte» sind) auch die Terminologie der wichtigsten Diagnoseund Therapiekodes (ICD und CHOP) kennen. Nur so können sie selbst später oder die Kodierer die entsprechenden Kodes abgreifen. Auch müssen Ärzte Grundkenntnisse in den schweizerischen Kodierregeln haben. Die Definitionen von Haupt- und Nebendiagnosen wie auch der Begriff des Ressourcenaufwandes müssen jedem Beteiligten klar sein. Als federführendes Beispiel sei hier erwähnt, dass gemäss aktuell geltendem Regelwerk die medizinische Hauptdiagnose nicht immer die zu kodierende Hauptdiagnose darstellt (gemäss Regelwerk ist die Diagnose die Hauptdiagnose, die den grössten Aufwand während des Spitalaufenthaltes generierte und am Ende des Spitalaufenthaltes feststeht).

\section{Anforderungen an die Ärzte}

- Kennt die Terminologie der wichtigsten Kodes (ICD und CHOP)

- Besitzt Grundkenntnisse der Schweizer Kodierregeln

- Erstellt für interdisziplinäre Patienten interdisziplinäre Berichte

\section{Anforderungen an}

\section{das medizinische Berichtswesen}

Berichte müssen in hochstandardisierter Form, aber auch sehr patientenindividuell abgefasst werden. Dies wurde bis anhin schweizweit so gehandhabt. Neu ist hier die «Nuance», dass die Sichtweise um die «Kodierungs- und Fakturierungstauglichkeit» erweitert wird. Eine grosse Erleichterung schafft die automatische Übernahme von administrativen Daten. Hierzu gehören u.a. Patientenname, Geburtsdatum, Wohnort, Ein- und Austrittsdatum, Aufenthaltsdauer, Adressat (Hausarzt) und vor allem die aufenthaltsspezifische Fallnummer. Nur die Fallnummer ist die «Identifikation» dieses Spitalaufenthaltes. Mit ihr lassen sich alle Informationen und Dokumente dieses Aufenthaltes zuordnen. Sie sollte auf keinem Dokument/Bericht fehlen.

\section{Anforderungen an das medizinische Berichtswesen \\ - Automatische Übernahme administrati- ver Patientendaten \\ - Automatische Übernahme der aufent- haltsbezogenen Fallnummer \\ - Stark standardisierter Aufbau}

Der Austrittsbericht richtet sich an unterschiedliche Teilnehmerkreise. Vor allen war und ist er auch weiterhin für den weiterbehandelnden Arzt bestimmt. Aber er muss auch juristischen und damit haftungsrechtlichen Anforderungen genügen. Mit der Einführung der DRGs als Finanzierungsmittel der Spitäler hat der Austrittsbericht eine weitere fundamentale Bedeutung erlangt. Er sollte all das enthalten, was den akutstationären Spitalaufenthalt unter Beachtung wichtiger Anforderungen der Kodierrichtlinien dokumentiert. Auch wichtige Informationen aus der Pflege wie Dekubitus, Harnwegsinfekt und Inkontinenz sollten im Austrittsbericht enthalten sein. Eine qualitativ hochwertige Kodierung ist dann möglich, wenn der Austrittsbericht Antworten auf folgende Fragen gibt: Was ist die Hauptdiagnose? Gibt es Nebendiagnosen? Wenn ja, waren diese behandlungsbedürftig? Was ist die Haupttherapie? Gibt es weitere Therapien? Werden anamnesti- 
sche Diagnosen (Diagnosen, die bei diesem stationären Aufenthalt nicht behandelt wurden) klar erkennbar hiervon abgegrenzt?

\section{Im Austrittsbericht bzw. internen Ver- laufsprotokoll enthaltene Informationen \\ - Angabe (Festlegung) der Hauptdiagnose (ICD) \\ - Nennung der Nebendiagnosen im Verlauf (ICD) \\ - Angabe der erfolgten Therapien (CHOP) \\ - Massnahmen auch aus dem Pflegebereich (Inkontinenz, Dekubitus, HWI usw.) \\ - Anamnestische Diagnosen (nicht Gegen- stand des jetzigen Aufenthalts) klar abge- grenzt}

Die chirurgischen Austrittsberichte betreffend, sei folgendes angemerkt: Chirurgische Komplikationen werden von Chirurgen korrekterweise meistens mit in den Bericht aufgenommen. Anders ist es hingegen mit internistischen oder neurologischen Nebendiagnosen auf Normalund Intensivstationen. Auch diese beeinflussen den aktuellen Aufenthalt (meist kostenintensive Behandlungen) und finden nur selten im Austrittsbericht des Chirurgen Berücksichtigung. Hier ist interdisziplinäre Kommunikation unter den Kollegen sinnvoll und auch erforderlich.

Auch unter Beachtung dieser Kriterien ist es möglich, den Bericht kurz zu halten. Es gilt der Grundsatz «Soviel wie nötig, aber sowenig wie möglich». Es kann durch die Erweiterung der Sichtweisen (Kodierung, Fakturierung über Diagnosen und Therapien) nicht sein, dass die Leistungserbringer mit mehr Dokumentationsaufwand belastet werden, sondern maximal mit einer präziseren, auch auf eine Finanzierung ausgerichteten Formulierung. Wir haben im Folgenden einen Vorschlag für einen standardisierten Austrittsbericht in Tabellenform beigefügt, der bereits in mehreren Spitälern praxiserprobt wurde. Dieses Muster in Tabellenform wurde mit Chef- und Leitenden Ärzten gemeinsam entwickelt. Er kann als Grundlage für alle Abteilungen verwendet und als Vorlage in der EDV hinterlegt werden. Die Struktur soll vor allem eine Hilfe und durch die Standardisierung eine wesentliche Entlastung für Assistenz- und Belegärzte sein. Damit kann auch bei wechselnden Ärzten eine gleichbleibende Qualität der Austrittsberichte erzielt werden.

\section{Die Quadratur des Kreises}

Der Austrittsbericht stellt mit Sicherheit hohe Anforderungen an den spitalinternen Prozess- ablauf. Es müssen Informationen aus unterschiedlichen Disziplinen berücksichtigt werden. Ebenso dürfen besondere Massnahmen aus Diagnostik, Pflege und Therapie nicht fehlen. Für eine korrekte Rechnungsstellung (DRG) sollten die ressourcenrelevanten Informationen mit abgebildet werden. Der weiterbehandelnde Arzt möchte eine kurze klinikbezogene Information erhalten, während der Kodierer hingegen Informationen zum Ressourcenverbrauch benötigt. Dies alles kurz in einem zusammenfassenden Dokument zu integrieren klingt wie die Quadratur des Kreises.

\section{Lösungswege}

Einen Königsweg gibt es sicherlich nicht. Hier muss jedes Spital unter Berücksichtung des zur Zeit Machbaren seinen eigenen Weg finden. Manche Spitäler generieren zwei Dokumente mit unterschiedlichen Schwerpunkten. Das eine Dokument ist für den weiterbehandelnden Arzt bestimmt und berücksichtigt die auf ihn zugeschnittene klinische Information in kurzer Form. Dies wäre dann der Austrittsbericht für den externen Kunden Arzt. Das andere Dokument ist für die behandelnden Ärzte der verschiedenen Disziplinen, die Pflege und auch für die Kodierkräfte bestimmt. Es enthält die klinischen Informationen des Therapieverlaufes in chronologischer Abfolge. Dieser Verlaufsbericht wird vom Assistenzarzt schon während des stationären Aufenthaltes als Ergebnis der Visiten kontinuierlich erstellt und dient zur Schnellinformation für Arzt und Pflege.

Es muss allerdings klar sein, dass die beiden Dokumente nicht unterschiedlich in den Aussagen sein sollen, sondern der Austrittsbericht hier lediglich eine Zusammenfassung des Verlaufsberichtes darstellt. Dies hat zusätzlich den Vorteil, dass auch längere Aufenthaltsdauern nachvollziehbar dargelegt werden können. Externer Austrittsbericht wie auch interner Verlaufsbericht sollten auf jeden Fall elektronisch abrufbar sein und sich klar erkennbar auf einen Aufenthalt beziehen.

In der Anfangsphase, also bis zur vollständigen Einführung einer elektronischen Krankenakte, ist dies sicher ein «gehbarer» Weg. Wir praktizieren ihn in unserem Spital mit grossem Erfolg.

\section{Organisationsvarianten der Kodierung}

Es gibt drei mögliche Varianten, eine Kodierung im Spital zu organisieren:

\section{Variante 1}

Der Kodierende ist der behandelnde Arzt selbst. 
Hierbei besteht der Vorteil, dass die Krankengeschichte dem «kodierenden Arzt» bekannt ist und er auf sein Wissen über seinen Patienten zurückgreifen kann. Der Nachteil bei dieser Variante ist, dass der behandelnde Arzt vertieft über alle relevanten Kodierregeln verfügen muss und seine eigentliche Dienstleistung, die Behandlung des Patienten, verlassen muss. Zudem ist der behandelnde Arzt als Kodierer mit z.T. massiven administrativen Tätigkeiten belastet.

\section{Variante 2}

Der Kodierende ist eine professionell ausgebildete Arbeitskraft mit der Grundausbildung Arzt, Fachkrankenpfleger Operationsabteilung oder/und Anästhesie oder diplomierte Pflege- kraft mit ausgeprägtem diagnostischem und therapeutischem Wissen. Zusätzlich verfügt er über den neuen Schweizer Nachdiplomkurs «eidg. dipl. Kodierer» oder «medizinischer Dokumentar» (aus Deutschland, jedoch «helvetisierte Weiterbildungen»). Hier ist der Vorteil, dass diese Arbeitskraft professionell in der Kodierung ausgebildet und tätig ist. Der Nachteil liegt auf der Hand. Er kennt den Patienten nicht «aus erster Hand», ist auf eine gute Dokumentation und Kommunikation angewiesen und hat allenfalls ein breitgefächertes Wissen, das fachlich «in der Tiefe» dem behandelnden Facharzt unterlegen ist. Eindeutig ist hier als Fazit zu nennen, dass diese Variante den behandelnden Arzt massiv von administrativen Tätigkeiten entlastet und der Professionalität in der Kodierung

Tabelle 1

Ablauf Berichtsstruktur und Hinweise.

\begin{tabular}{|c|c|}
\hline Ablauf Berichtsstruktur & Hinweise \\
\hline Patientendaten & $\begin{array}{l}\text { Name, Vorname, Geb.-Datum, Adresse, Aufenthaltsdauer, } \\
\text { Fallnummer }\end{array}$ \\
\hline Hospitalisation & vom ... bis ... \\
\hline \multicolumn{2}{|c|}{$\begin{array}{l}\text { Beispiel: } \\
\text { Egon Mustermann, geb. 20.12.1976, Vordergasse 16, } 5620 \text { Bremgarten, Fall-Nr.: } 1234567 \\
\text { Stationär vom: 23.4. bis 4.5.2007. }\end{array}$} \\
\hline \multicolumn{2}{|l|}{ Eintrittsgrund/Zuweisungsgrund } \\
\hline Beschreibung der Symptome, Angabe Unfallhergang & $\begin{array}{l}\text { Kurze Beschreibung des Geschehens, warum der Patient } \\
\text { ins Spital kommt. Gibt Hinweise zum Grund des Spital- } \\
\text { aufenthaltes: ist oft (nicht immer!) die Hauptdiagnose }\end{array}$ \\
\hline \multicolumn{2}{|c|}{ Diagnosen (an erster Stelle Hauptdiagnose, ab der 2. Stelle die Nebendiagnosen) } \\
\hline Hauptdiagnose & $\begin{array}{l}\text { Hauptdiagnose ist jene Diagnose, die den meisten } \\
\text { Aufwand verursacht hat und den Hauptgrund der } \\
\text { Behandlung darstellt (zum Zeitpunkt des Austritts) }\end{array}$ \\
\hline Nebendiagnosen & $\begin{array}{l}\text { Nebendiagnosen sind Diagnosen, die Mehraufwand } \\
\text { in der Behandlung oder einen längeren Aufenthalt } \\
\text { verursachen }\end{array}$ \\
\hline \multicolumn{2}{|c|}{$\begin{array}{l}\text { Beispiel: } \\
\text { Hauptdiagnose: 1. Diarrhoe unklarer Genese } \\
\text { Nebendiagnosen: 2. Arterielle Hypertonie, 3. Struma nodosa linksbetont } \\
\text { Anamnest. Diagnosen: } 4 \text {. St. n. Aortenklappenersatz 1983, 5. Coxarthrose links }\end{array}$} \\
\hline Histologie & Gibt präzise Hinweise zur Hauptdiagnose \\
\hline \multicolumn{2}{|l|}{ Für Chirurgie } \\
\hline \multicolumn{2}{|l|}{$\begin{array}{l}\text { Beispiel: } \\
\text { 1. Nekroseexzision tief rechte Ferse am 4.2.2006 } \\
\text { 2. Wundkonditionierung mit VAC-Pumpe vom 6.2. bis } 15.2 \\
\text { 3. Antibiotische Behandlung mit Augmentin vom 6.2. bis } 15 \\
\text { 4. Unterschenkelamputation rechts am 15.2.2006 } \\
\text { 5. Wunddébridement und Fadenentfernung am 6.3.2006 }\end{array}$} \\
\hline \multicolumn{2}{|l|}{ Für Medizin } \\
\hline Verlauf & $\begin{array}{l}\text { Beschreibung der Haupt- und jeder Nebendiagnose: } \\
\text { ad } 1 . / \text { ad } 2 . / \text { ad } 3 \text {. usw. }\end{array}$ \\
\hline Prozedere & Weiteres geplantes Vorgehen \\
\hline Medikation bei Austritt & Name (ggf. Wirkstoff), Dosierung \\
\hline
\end{tabular}


(durch eine hauptamtliche Tätigkeit von ausgebildeten Kodierern) den Weg ebnet.

\section{Variante 3}

Diese Variante besteht aus einer Kombination der Varianten 1 und 2. Hierbei kodieren die Ärzte selbst, und der Kodierer arbeitet als «Kodier-Controller», indem er die kodierten Fälle der behandelnden Ärzte mit den vorhandenen und gültigen Kodierrichtlinien abgleicht, allenfalls Korrekturen im Sinne der Regeln in Absprache mit dem Arzt vornimmt. Zusätzlich gibt er die kodierten Daten in den Grouper und ins Administrativsystem ein und plausibilisiert den Fall. Der Vorteil ist bei dieser Variante, dass eine «doppelte Sicherheit» in der Kodierung entstehen kann und eine vertiefte Kommunikation zwischen ärztlichem Dienst und Administration (Kodierung) stattfindet. Der Nachteil liegt in der «Gewaltentrennung» auf zwei Abteilungen (Arzt und Kodierer), die sehr grossen administrativen Aufwand generiert und gegenüber den Varianten 1 und 2 mit Abstand am teuersten zu sein scheint.

Grundsätzlich kann man nur nach den vorhandenen Strukturen des jeweiligen Spitals die eine oder andere Variante empfehlen. Gemäss unserer Erfahrung ist die Variante 2 die am meisten verbreitete in den Schweizer Spitälern. Diese Variante wird mit grossem Erfolg auch in unserem Spital unter der Leitung eines Arztes als Medizincontroller eingesetzt.

\section{Anforderungen an den finanzrelevanten Kodierprozess}

Der Kodierer (in diesem Falle gehen wir von der schweizweit am meisten angewandten Variante 2 aus) sitzt meistens am Ende der Informationskette. Er kennt den Patienten (wie oben beschrieben) nicht und muss nach Aktenlage entscheiden, weil er den Patienten nicht selbst behandelt hat. Seine Tätigkeit kann also nur so gut sein, wie die ihm zur Verfügung gestellten Informationen dies auch hergeben. Entscheidend ist hierbei, dass der Kodierer keinerlei Informationen selbst generiert, sondern seine Tätigkeit, der Kodierprozess, lediglich das «Abgreifen» von vorhandenen Informationen darstellt. Versteckte oder unleserliche Informationen in der Krankengeschichte sind für die Kodierung unbrauchbar, oftmals findet der Kodierende diese erst gar nicht. Der Kodierprozess wird unnötig verlängert, und kodierrelevante Informationen werden nicht gefunden. Der Kodieraufwand wird verteuert. Gleichzeitig ist der klinische Aufenthalt oftmals in der Folge mit einer Erlösminderung verbunden.

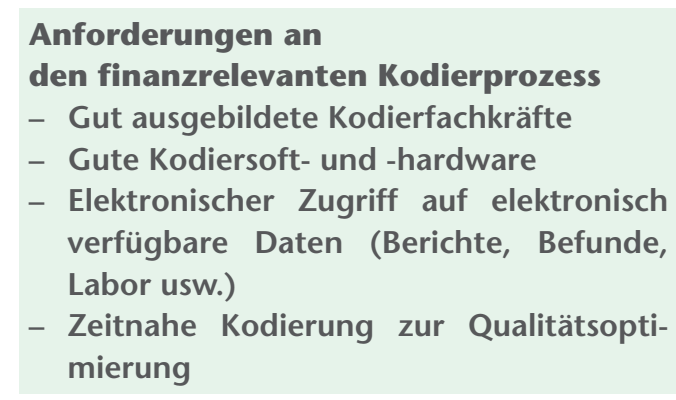

Der Kodierer benötigt für seine Arbeit professionelle Hard- und Softwaretools. Das Arbeiten an zwei Bildschirmen gleichzeitig kann hierbei sehr effizient eingesetzt werden. Eine ausgereifte Kodiersoftware mit Einbindung eines für die Schweiz zertifizierten Groupers ist unumgänglich. Patientensuche nach Fallnummer, Name, Geburtsdatum, Aufenthaltsdatum und Fachabteilung sollte ebenso möglich sein wie das Erstellen von Listen für noch $\mathrm{zu}$ kodierende Fälle. Je mehr Informationen elektronisch zur Verfügung stehen, um so schneller, leichter und vor allem korrekter wird die Kodiertätigkeit und somit die Rechnungsstellung. Labordaten wie auch zusammenfassende Dokumente (Befunde, OP-Berichte, Konsile, Austrittsberichte) sollten elektronisch zeitnah zur Verfügung stehen.

Die Effizienz des gesamten Dokumentationsund Kodierprozesses steigt mit der zeitnahen Kodierung. Nur bei einer zeitnahen Kodierung ist bei Rückfragen das Erinnerungsvermögen der Prozessbeteiligten (Arzt, Pflege, Sozialdienst usw.) noch gut abgreifbar. Schwachstellen in der Dokumentation können leichter erkannt und verbessert werden. Überprüfungen der Aufenthaltsdauer können mit dem Ziel der Verbesserung klinikinterner Abläufe kontinuierlich zum Beispiel in Form des Peer-Review-Verfahrens - erfolgen.

Nur der kodierte Fall ermöglicht ein DRGBerichtswesen als Führungs- und Steuerungsinstrument klinischer Leistungen.

Nur die zeitnahe Kodierung ermöglicht, Qualität messbar zu machen und die Prozesse stetig an den Messergebnissen zu verbessern.

Nur die professionelle Organisation der Kodierung entlastet den ärztlichen Leistungserbringer von «lästigen» administrativen Tätigkeiten.

Die Kodierung sollte einen hohen Stellenwert im «Unternehmen Spital» haben. Schliesslich generieren die Kodierer die DRGs, den Case-Mix (CM) und den Case-Mix-Index (CMI) sowie die Faktura über APDRG, die für die Kostenträger (Versicherer und Kantone) die grundlegende Basis für Budgetverhandlungen und Erlöse darstellen. 


\section{Commentaire de la FMH}

Les auteurs de cet article nous rendent attentifs à toute une série de problèmes réels. Le dogmatisme des solutions proposées interpelle cependant les médecins hospitaliers et amène certaines remarques de la part du corps médical:

- Le respect de la protection des données est primordial. L'idée d'avoir un dossier entièrement compréhensible par un tiers laisse supposer des dérives possibles dans ce domaine. D'autre part, la présence d'indications sensibles dans un système informatique implique un risque énorme de diffusion à des personnes non autorisées, comme les utilisateurs de dossiers électroniques ont déjà pu le constater. Le développement d'un tel système nécessite donc un strict contrôle d'accessibilité avec des codes d'entrée sécurisés déjà à l'intérieur de l'hôpital.

- La diffusion de données médicales à l'extérieur de I'hôpital doit se limiter à la lettre de sortie destinée aux médecins impliqués. Elle peut être faite par voie électronique mais devrait, dans ce cas, transiter par un système de transmission hautement sécurisé (tel que HIN par exemple). La diffusion de données médicales aux assureurs n'est pas acceptable en dehors de la voie du médecin-conseil. Un filtrage serré des demandes incessantes des assureurs est à prévoir, afin d'éviter toute transmission de données médicales plus ou moins exhaustives à des instances administratives non autorisées.

- La lettre de sortie est le document médical central d'un séjour hospitalier. Si elle est en premier lieu destinée au médecin traitant, elle sert également de conclusion, à l'interne, pour toute la démarche diagnostique et thérapeutique effectuée. A ce titre, elle nécessite une réflexion de synthèse et ne saurait être une juxtaposition d'éléments chronologiques. Ceci n'empêche nullement qu'elle soit exhaustive, en particulier qu'elle contienne toutes les indications pertinentes pour le codage: I'inclusion de ces précisions fait justement partie de la réflexion de celui qui crée le document. Si ce travail est effectué correctement sous le contrôle systématique du médecin-cadre responsable et que des notes de suite sont régulièrement inscrites par les assistants, il n'y a pas lieu de créer un document séparé à usage interne.

- Quelle que soit l'organisation du codage, le médecin doit en garder le contrôle et la responsabilité. Les éventuels codificateurs doivent en référer au médecin-cadre responsable pour tous les cas peu clairs ou difficiles.

- La structure tarifaire SwissDRG sera appliquée à tout le pays. Le système APDRG sera alors abandonné. Par conséquent, toute réflexion au sujet de mesures consécutives à l'introduction de SwissDRG ne devrait plus faire référence à APDRG.

- SwissDRG s'appuiera sur les classifications ICD et CHOP. Cette dernière sera probablement adaptée et augmentée. Cependant, elle contiendra toujours des procédures diagnostiques et thérapeutiques susceptibles d'influencer le poids relatif des coûts lors du groupage. Comme ces procédures sont distinctes des interventions, elles ne doivent pas être oubliées afin d'être saisies. Elles peuvent également être mises en évidence dans la lettre de sortie.

- Le langage médical est une entité précise bien définie qui s'est développée au cours des siècles de la pratique de notre art. Au même titre que nous ne saurions sacrifier notre liberté thérapeutique sur l'autel des pressions économiques, il est hors de question d'adapter nos expressions au moule des manuels de codage et des groupeurs, apparitions éphémères qui, pour être performantes, doivent être à même de comprendre notre terminologie.

Dr P.-F. Cuénoud, membre du Comité central de la FMH, préposé SwissDRG 